\title{
Diversidad y estructura genética de una población de cabras criollas negras de tres municipios del estado de Querétaro, México
}

\section{Juan Carlos Silva-Jarquin ${ }^{\text {a }}$}

Héctor Mario Andrade-Montemayor ${ }^{b *}$

Héctor Raymundo Vera-Ávila ${ }^{\mathrm{b}}$

Marina Durán-Aguilar ${ }^{b}$

Sergio Iván Román-Ponce ${ }^{c}$

Vincenzo Landi ${ }^{\mathrm{d}}$

Amparo Martínez-Martínez ${ }^{\mathrm{d}}$

Juan Vicente Delgado Bermejo ${ }^{\mathrm{d}}$

Consorcio BioGoat ${ }^{\mathrm{e}}$

${ }^{\text {a }}$ Universidad Autónoma de Querétaro. Facultad de Ciencias Naturales. Doctorado en Ciencias Biológicas. Avenida de las Ciencias S/N Juriquilla, Delegación Santa Rosa Jáuregui, 76230 Querétaro, México.

${ }^{\mathrm{b}}$ Universidad Autónoma de Querétaro. Facultad de Ciencias Naturales. Licenciatura en Medicina Veterinaria y Zootecnia. México.

${ }^{c}$ INIFAP, Centro Nacional de Investigación Disciplinaria en Fisiología y Mejoramiento Animal. México.

d Universidad de Córdoba. Campus de Excelencia Internacional Agroalimentario ceiA3. Departamento de Genética. España.

e Proyecto de Biodiversidad Caprina Iberoamericana. España.

*Autor de correspondencia: andrademontemayor@gmail.com 


\section{Resumen:}

Desde su llegada a México, las cabras han experimentado un largo proceso de adaptación y selección, resultando animales locales de elevada rusticidad. Sin embargo, la importación de razas mejoradas, ha inducido la extinción de algunas razas. Por ejemplo, la cabra criolla negra $(\mathrm{CCN})$, reconocida por su rusticidad y la calidad de su leche. El objetivo del presente trabajo fue caracterizar genéticamente una población de CCN. Se colectaron muestras de pelo en tres rebaños caprinos ubicados en diferentes municipios del estado de Querétaro; Cadereyta de Montes ( $n=7)$, El Marqués ( $n=11)$ y San Juan del Río ( $\mathrm{n}=27$ ). Se utilizaron 30 microsatélites, obteniendo; el número de alelos por marcador (NA), el número medio de alelos (NMA), número efectivo de alelos (NEA), la heterocigosis observada (Ho) y esperada (He), el contenido de información polimórfica (CIP), el índice de fijación (FIS) y el equilibrio de Hardy Weinberg (EHW). La población se comparó con 13 razas del proyecto BioGoat. Los resultados mostraron que existe una elevada diversidad genética en este ganado. Se obtuvieron 243 alelos con un NMA de 8.1 alelos por marcador. Los marcadores resultaron informativos $(\mathrm{CIP}=0.06)$ respecto a su polimorfismo. La He (0.71) y Ho (0.62) indican que existe un ligero desequilibrio en la población. La distancia de Reynolds mostró que la $\mathrm{CCN}$ se encuentra más distanciada genéticamente de la población Anglonubia y más cercana a la Murciano-Granadina. Los resultados aquí presentados sugieren que la población $\mathrm{CCN}$ representa una estructura racial bien diferenciada de las poblaciones incluidas en el estudio.

Palabras clave: Caracterización genética, Genética de poblaciones, Cabra criolla negra.

Recibido: 19/05/2018

Aceptado: 22/10/2018

\section{Introducción}

Desde el inicio del proceso de domesticación, hace 10,000 años aproximadamente, el ganado caprino ha estado en estrecha relación con la especie humana ${ }^{(1,2)}$. Se considera uno de los primeros animales de granja en ser domesticado y ha participado en acontecimientos como la revolución agrícola del Neolítico, el desarrollo del comercio y las migraciones humanas ${ }^{(3)}$. Estos acontecimientos implicaron algunos mecanismos básicos de la evolución, tales como; migración de animales, selección, deriva génica e incluso mutación, lo que ayuda a explicar en gran medida la elevada capacidad de adaptación del ganado caprino a diferentes ecosistemas y las más de 300 razas que existen en la actualidad $^{(1,4)}$. 
Desde su llegada al continente americano en 1493, los colonizadores españoles se encargaron de propagar la especie caprina, así como de capacitar a pobladores nativos en su manejo y en la selección de los mejores animales. Con el paso del tiempo, estas acciones dieron origen a nuevas poblaciones, las cuales estaban mejor adaptadas a las condiciones ambientales locales; poblaciones denominadas criollas ${ }^{(5)}$.

Actualmente en México existen poco más de 8.7 millones de cabras ${ }^{(6)}$, y al igual que en otros países en vías de desarrollo, éstas representan una oportunidad de subsistencia para las personas que habitan en zonas áridas y semiáridas, donde la vegetación es escasa y las condiciones del agostadero son pobres ${ }^{(7,8)}$. Aun cuando se distribuyen por todo el país, se han agrupado en tres regiones principales; el mosaico mixteco, el del centro o bajío y el del norte o lagunero ${ }^{(9)}$. Particularmente, en el mosaico del centro del país se encuentra la Cabra Criolla Negra (CCN), la cual se distribuye principalmente en los estados de Querétaro y Guanajuato. La principal finalidad zootécnica de la CCN es la producción de leche, la cual presenta un mayor contenido de sólidos totales y un excelente rendimiento quesero con respecto a otras razas criadas en México ${ }^{(10,11,12)}$. Esta cabra era considerada como raza Granadina por sus características morfológicas y por su origen, sin embargo, los más de 500 años de evolución independiente han hecho que se diferencie genéticamente de esta raza. Pese lo anterior, no existen trabajos previos que estudien el estado genético de la CCN. Las importaciones de animales de razas mejoradas se han incrementado en los últimos años y se vienen realizando cruzamientos de forma indiscriminada, lo que pone en riesgo el estado de salud genética de la población ${ }^{(13)}$.

La evaluación de la diversidad genética dentro y entre las razas permite conocer la estructura de la población y hace posible establecer estrategias de conservación, mejoramiento genético y utilización sostenible de los recursos genéticos ${ }^{(14)}$. Los marcadores moleculares del tipo microsatélite han demostrado ser de gran utilidad para los estudios de caracterización genética dentro y entre poblaciones, esto debido a su codominancia genética, abundancia, distribución aleatoria a través del genoma, alta reproducibilidad, neutralidad con respecto a la selección y alto nivel de polimorfismo $^{(14,15)}$. En los últimos años, se han llevado a cabo numerosos estudios de diversidad genética en varias especies de ganado utilizando estos marcadores, convirtiéndose en los marcadores genéticos de elección para muchas aplicaciones moleculares, algunas de estas son: diversidad genética ${ }^{(16,17)}$, estructura poblacional ${ }^{(18,19)}$, filogenia ${ }^{(20)}$, evaluación de paternidad ${ }^{(21)}$, etc. El objetivo del presente estudio fue evaluar la diversidad genética y la estructura poblacional de la Cabra Criolla Negra a través de marcadores microsatélites. 


\section{Material y métodos}

\section{Muestras biológicas}

Se colectaron muestras de pelo de 45 animales pertenecientes a tres rebaños de CCN utilizando el método no probabilístico de oportunidad y ubicados en tres municipios del estado de Querétaro; Cadereyta de Montes (n=7), El Marqués ( $\mathrm{n}=11)$ y San Juan del Río $(n=27)$. Los animales se muestrearon utilizando el siguiente criterio de inclusión; animales no emparentados, mayores a un año de edad, de capa negra con orejas rígidas o semipendulantes. En estas poblaciones no se cuenta con información genealógica y debido a esto se tomó en cuenta la información de parentesco entre los animales proporcionada por los propietarios.

Se incluyó la información de 25 microsatélites localizados en 455 individuos de 13 poblaciones caprinas (Retinta, Verata, Blanca Serrana, Celtibérica, Malagueña, Murciano-Granadina, Florida, Payoya, Serrana, Formentera, Saanen, Alpina y Anglonubia) integradas en el proyecto de Biodiversidad Caprina Iberoamericana (BioGoat) ${ }^{(22)}$.

\section{Análisis molecular}

La extracción del ADN se realizó a partir de las muestras de pelo utilizando resina quelante Chelex ${ }^{\circledR} 100$ (Bio Rad Laboratories, Inc. USA) ${ }^{(23)}$. Se utilizaron 30 microsatélites recomendados por el comité mixto ISAG/FAO para el análisis de la diversidad genética en animales domésticos ${ }^{(14)}$ de los cuales, 25 marcadores se encontraron en común con las 13 poblaciones utilizadas del proyecto BioGoat. La amplificación de los marcadores se realizó por reacción en cadena de la polimerasa (PCR), para lo cual se utilizaron cebadores marcados con fluorescencia ${ }^{(24)}$. Los amplicones obtenidos como resultado de la PCR fueron separados por electroforesis capilar (ABI PRISM 3130 Genetic Analyser; Applied Biosystems) de acuerdo con las recomendaciones del fabricante. El tamaño de los alelos se determinó utilizando el estándar de tamaño interno GeneScan-400HD ROX (Applied Biosystems) mientras que los genotipos fueron obtenidos por medio del software GENOTYPER 2.5.1. Se incluyeron muestras de referencia en cada prueba como método de comprobación del genotipado. 


\section{Análisis estadístico}

El número total de alelos por marcador (NA) se calculó por conteo directo de los alelos obtenidos para cada uno de estos, mientras que, el número medio de alelos (NMA) se calculó como la sumatoria del NA dividida entre los 30 marcadores utilizados. La heterocigosis observada $\left(\mathrm{H}_{\mathrm{o}}\right)$ fue resultado de dividir el número de individuos heterocigotos en cada marcador entre el total de individuos positivos al mismo marcador. La heterocigosis esperada $\left(\mathrm{H}_{\mathrm{e}}\right)$ se estimó utilizando la fórmula de $\mathrm{Nei}^{(25)}$. El contenido de información polimórfica (CIP) que representa un indicativo de la calidad de los marcadores utilizados $^{(26)}$ se estimó utilizando el complemento para Microsoft Excel 2010 MICROSATELLITE TOOLKIT ${ }^{(27)}$. El número efectivo de alelos (NEA), el cual hace referencia a los alelos con capacidad de pasar a la siguiente generación ${ }^{(28)}$, se realizó a través del software POPGENE v. 1.32. La prueba exacta del equilibrio Hardy-Weinberg, misma que considera el déficit de heterocigotos se realizó mediante el software GENEPOP v.4.2(29). Esta prueba se llevó a cabo para los 30 marcadores utilizados en la CCN ocupando el método en cadena de Markov (desmemorización 5,000, 100 lotes y 10,000 interacciones por lote).

El coeficiente de endogamia de los individuos respecto a las subpoblaciones ( $F_{I S}$ ), respecto al total de la población $\left(\mathrm{F}_{\mathrm{IT}}\right)$, de las subpoblaciones comparado con el total de la población $\left(\mathrm{F}_{\mathrm{ST}}\right)$ y el coeficiente de diferenciación genética $\left(\mathrm{G}_{\mathrm{ST}}\right)$ se calcularon con un intervalo de confianza de $95 \%^{(25,30)}$. La estimación de estos parámetros se realizó mediante el software GENETIX v. $4.05^{(31)}$.

Se calculó la matriz de distancias genéticas de Reynolds ${ }^{(32)}$ que es la distancia mínima de Nei normalizada con una valoración de heterocigosis en la población fundadora utilizando el software POPULATIONS v.1.2.28. Se obtuvieron Split Graphs mediante el algoritmo "NeighborNet" utilizando el programa SPLITSTREE4 ${ }^{(33)}$.

El origen de la estructura genética de las poblaciones incluidas en el estudio se determinó mediante técnicas de clúster $(\mathrm{K})$, que representan el número de poblaciones y usa un algoritmo bayesiano que emplea un modelo basado en el método de Cadenas de Markov de Monte Carlo (CMMC), que estima la distribución a posteriori de cada coeficiente de mezcla de cada individuo, el cual se realizó utilizando el software STRUCTURE v.2.3.4 ${ }^{(34)}$. Se utilizó un periodo de "burn-in" de 50,000 iteraciones y 200,000 repeticiones de CMMC, los resultados se visualizaron mediante el programa DISTRUCT ${ }^{(35)}$. Para estimar el K óptimo, se fijaron valores de K2 a K15, el análisis se realizó con 15 repeticiones para cada valor de K siguiendo la metodología de Evanno ${ }^{(36)}$ y utilizando el software STRUCTURE HARVESTER ${ }^{(37)}$. 


\section{Resultados}

\section{Variación genética en la población Criolla Negra}

Se obtuvieron 243 alelos para los 30 marcadores utilizados. El NMA fue de 8.1 alelos por locus en esta población (Cuadro 1). El NA más alto por marcador (13) lo presentaron MM12 y SRCRSP23, seguido por los marcadores BM6526 y HSC con 12 alelos. El marcador con menor NA fue MAF209 con 2 alelos. Adicionalmente, el NEA con mayor valor correspondió a HSC $(\mathrm{NEA}=9.14)$ y el de menor valor fue para MAF209 (NEA= 1.25), este resultado pudiera verse afectado por la proporción de marcadores polimórficos, el número de alelos por marcador y sus frecuencias, así como por el tamaño de la muestra. La heterocigosis esperada (He) promedio en la población fue de 0.71 , este valor varió entre 0.20 para MAF209 y 0.90 para HSC. El promedio de heterocigosis observada (Ho) fue de 0.62 y el rango 0.18 y 0.93 para los mismos marcadores que mostraron valores extremos para He.

Cuadro 1: Microsatélites analizados, número de alelos detectados (NA), número efectivo de alelos (NEA), heterocigosis esperada (He) y observada (Ho), contenido de información polimórfica (CIP) y las desviaciones del equilibrio Hardy-Weinberg (EHW)

\begin{tabular}{lllllll}
\hline Microsatélite & NA & NEA & He & Ho & CIP & $\begin{array}{l}\text { EHW } \\
\text { P-val }\end{array}$ \\
\hline BM1329 & 8 & 3.44 & 0.72 & 0.67 & 0.68 & 0.20 \\
BM1818 & 8 & 4.63 & 0.79 & 0.71 & 0.76 & 0.12 \\
BM6506 & 9 & 3.26 & 0.70 & 0.55 & 0.66 & 0.02 \\
BM6526 & 12 & 4.93 & 0.81 & 0.86 & 0.78 & 0.90 \\
BM8125 & 6 & 3.64 & 0.73 & 0.62 & 0.68 & 0.02 \\
CRSM60 & 8 & 4.32 & 0.78 & 0.71 & 0.73 & 0.03 \\
CSRD247 & 6 & 3.25 & 0.70 & 0.77 & 0.66 & 0.97 \\
CSSM66 & 11 & 7.06 & 0.87 & 0.34 & 0.84 & 0.00 \\
ETH010 & 4 & 2.69 & 0.63 & 0.56 & 0.56 & 0.19 \\
ETH225 & 4 & 1.41 & 0.29 & 0.25 & 0.26 & 0.22 \\
HAUT27 & 8 & 4.11 & 0.77 & 0.71 & 0.72 & 0.28 \\
HSC & 12 & 9.14 & 0.90 & 0.93 & 0.88 & 0.31 \\
ILSTS011 & 6 & 2.87 & 0.66 & 0.59 & 0.59 & 0.33 \\
INRA063 & 5 & 2.46 & 0.60 & 0.64 & 0.51 & 0.72 \\
MAF065 & 11 & 5.78 & 0.84 & 0.87 & 0.81 & 0.24 \\
MAF209 & 2 & 1.25 & 0.20 & 0.18 & 0.18 & 0.43 \\
McM527 & 8 & 5.33 & 0.82 & 0.82 & 0.79 & 0.58 \\
MM12 & 13 & 7.00 & 0.87 & 0.75 & 0.84 & 0.03
\end{tabular}




\begin{tabular}{lllllll} 
OarFCB011 & 10 & 5.73 & 0.83 & 0.75 & 0.80 & 0.12 \\
OarFCB048 & 10 & 7.14 & 0.87 & 0.78 & 0.84 & 0.01 \\
OarFCB304 & 10 & 3.72 & 0.74 & 0.61 & 0.69 & 0.00 \\
SPS115 & 3 & 2.02 & 0.51 & 0.27 & 0.44 & 0.00 \\
SRCRSP08 & 10 & 3.76 & 0.74 & 0.70 & 0.69 & 0.23 \\
TGLA122 & 8 & 2.20 & 0.55 & 0.49 & 0.52 & 0.02 \\
SRCRSP05 & 7 & 3.09 & 0.68 & 0.59 & 0.63 & 0.05 \\
SRCRSP23 & 13 & 8.49 & 0.89 & 0.68 & 0.87 & 0.00 \\
SRCRSP24 & 10 & 3.59 & 0.73 & 0.56 & 0.69 & 0.00 \\
ILSTS019 & 6 & 2.27 & 0.57 & 0.51 & 0.53 & 0.04 \\
INRA005 & 5 & 2.23 & 0.56 & 0.45 & 0.51 & 0.03 \\
INRA006 & 10 & 5.29 & 0.82 & 0.77 & 0.79 & 0.18 \\
Promedio & 8.1 & 4.20 & 0.71 & 0.62 & 0.66 & \\
\hline
\end{tabular}

$P>0.05=$ No significativo

El valor promedio del CIP para la población de CCN fue de 0.66. En este caso, el marcador menos informativo (CIP <0.25) fue MAF209 ( CIP=0.18), seguido de ETH225 y SPS 115 con valores de 0.26 y 0.44 , mientras que los 27 marcadores restantes fueron más informativos (CIP >0.5). Finalmente, se observaron desviaciones significativas del equilibrio de Hardy Weinberg (EHW) en 14 de los 30 microsatélites probados en la población $(P \leq 0.05)$.

\section{Diferenciación genética entre poblaciones}

El coeficiente de endogamia ( $F_{\text {IS }}, F_{\text {IT }}$ y $F_{\text {ST }}$ ) y el coeficiente de diferenciación genética (GST) se estimaron para cada uno de los 25 microsatélites compartidos entre la CCN y las otras 13 razas incluidas en el proyecto BioGoat ${ }^{(22)}$ con un intervalo de confianza del $95 \%$ (Cuadro 2). El valor promedio para FIs fue de 0.067 , presentando valores negativos de FIS en los marcadores BM8125 y MAF209 (-0.002 y -0,006) indicando en estos un exceso de heterocigotos ${ }^{(18,19)}$. De los marcadores utilizados 11 presentaron un valor de FIs superior a 0.05. Los valores de $\mathrm{G}_{\mathrm{ST}}$ fueron similares a los obtenidos para, FST. El marcador que representa un valor más alto fue BM6526 (0.114), en contraste con el MAF209 que fue el más bajo (0.037). 
Cuadro 2: Coeficiente de diferenciación genética y coeficiente de endogamia para cada microsatélite compartido entre la cabra criolla negra y las razas Retinta, Verata, Blanca serrana, Celtibérica, Malagueña, Murciano granadina, Florida, Payoya, Serrana,

Formentera, Saanen, Alpina, Anglonubia

\begin{tabular}{lllll}
\hline Microsatélite & GST & F & F & F \\
\hline BM1329 & 0.081 & 0.024 & 0.078 & 0.056 \\
BM1818 & 0.077 & 0.024 & 0.085 & 0.062 \\
BM6506 & 0.074 & 0.044 & 0.094 & 0.053 \\
BM6526 & 0.114 & 0.045 & 0.111 & 0.069 \\
BM8125 & 0.076 & -0.002 & 0.065 & 0.067 \\
CRSM60 & 0.041 & 0.064 & 0.091 & 0.029 \\
CSRD247 & 0.082 & 0.026 & 0.095 & 0.071 \\
CSSM66 & 0.083 & 0.271 & 0.316 & 0.062 \\
ETH010 & 0.058 & 0.032 & 0.076 & 0.047 \\
ETH225 & 0.057 & 0.015 & 0.058 & 0.044 \\
HSC & 0.068 & 0.095 & 0.141 & 0.050 \\
ILSTS011 & 0.058 & 0.068 & 0.114 & 0.049 \\
INRA063 & 0.051 & 0.164 & 0.195 & 0.038 \\
MAF065 & 0.073 & 0.025 & 0.084 & 0.060 \\
MAF209 & 0.037 & -0.006 & 0.018 & 0.024 \\
McM527 & 0.081 & 0.087 & 0.151 & 0.070 \\
MM12 & 0.060 & 0.059 & 0.098 & 0.042 \\
OarFCB011 & 0.084 & 0.065 & 0.134 & 0.073 \\
OarFCB048 & 0.060 & 0.058 & 0.103 & 0.049 \\
SPS115 & 0.097 & 0.187 & 0.257 & 0.086 \\
SRCRSP08 & 0.106 & 0.049 & 0.143 & 0.099 \\
TGLA122 & 0.091 & 0.070 & 0.146 & 0.082 \\
Promedio & 0.073 & 0.067 & 0.121 & 0.058 \\
\hline FS=coeficin & \\
\hline
\end{tabular}

$\mathrm{F}_{\mathrm{IS}}=$ coeficiente de endogamia de los individuos respecto a las subpoblaciones, $\mathrm{F}_{\mathrm{IT}}=$ coeficiente de endogamia respecto al total de la población, $\mathrm{F}_{\mathrm{ST}}=$ coeficiente de endogamia de las subpoblaciones comparado con el total de la población, $\mathrm{G}_{\mathrm{ST}}=$ coeficiente de diferenciación genética.

\section{Distancia genética entre poblaciones y su representación gráfica}

Las distancias genéticas entre las 14 razas comparadas se presentan en el Cuadro 3. La menor distancia genética se presentó entre la CCN y la raza Murciano-Granadina (0.133). La mayor distancia genética se obtuvo con la raza Anglonubia (0.420). Con el objetivo 
de facilitar la interpretación de los valores obtenidos en la matriz de distancias genéticas, se construyó un dendrograma Neighbor-Net para representar gráficamente estos valores (Figura 1).

Cuadro 3: Matriz de distancias genéticas de Reynolds entre las 14 razas caprinas incluidas en el estudio

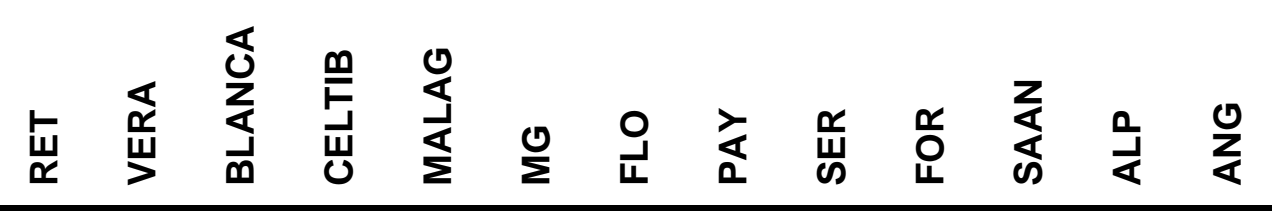

VERA $\quad 0.025$

BLANCA $\quad 0.025 \quad 0.032$

CELTIB $\quad 0.025 \quad 0.036 \quad 0.027$

$\begin{array}{lllll}\text { MALAG } \quad 0.021 & 0.025 & 0.023 & 0.021\end{array}$

$\begin{array}{llllll}\text { MG } & 0.044 & 0.045 & 0.031 & 0.034 & 0.041\end{array}$

$\begin{array}{lllllll}\text { FLO } & 0.023 & 0.023 & 0.028 & 0.026 & 0.015 & 0.041\end{array}$

$\begin{array}{llllllll}\text { PAY } & 0.034 & 0.044 & 0.047 & 0.036 & 0.046 & 0.061 & 0.038\end{array}$

$\begin{array}{lllllllll}\text { SER } & 0.034 & 0.038 & 0.035 & 0.028 & 0.024 & 0.048 & 0.027 & 0.045\end{array}$

$\begin{array}{llllllllll}\text { FOR } & 0.071 & 0.094 & 0.081 & 0.078 & 0.083 & 0.099 & 0.083 & 0.081 & 0.107\end{array}$

$\begin{array}{lllllllllll}\text { SAAN } & 0.071 & 0.061 & 0.070 & 0.069 & 0.070 & 0.069 & 0.061 & 0.077 & 0.078 & 0.119\end{array}$

$\begin{array}{llllllllllll}\text { ALP } & 0.063 & 0.056 & 0.052 & 0.062 & 0.055 & 0.076 & 0.049 & 0.064 & 0.073 & 0.119 & 0.071\end{array}$

$\begin{array}{lllllllllllll}\text { ANG } & 0.124 & 0.125 & 0.130 & 0.151 & 0.132 & 0.146 & 0.126 & 0.178 & 0.149 & 0.221 & 0.161 & 0.142\end{array}$

$\begin{array}{llllllllllllll}\text { CCN } & 0.039 & 0.052 & 0.045 & 0.044 & 0.046 & 0.038 & 0.048 & 0.067 & 0.053 & 0.102 & 0.083 & 0.073 & 0.130\end{array}$

RET = Retinta, VERA= Verata, BLANCA= Blanca serrana, CELTIB= Celtibérica, MALAG= Malagueña, MG= Murciano granadina, FLO= Florida, $\mathrm{PAY}=$ Payoya, $\mathrm{SER}=$ Serrana, FOR= Formentera, SAAN= Saanen, ALP= Alpina, ANG= Anglonubia, CCN= Criolla negra. 
Figura 1: Dendrograma Neighbor-Net construido con la distancia genética de Reynolds entre 14 razas caprinas

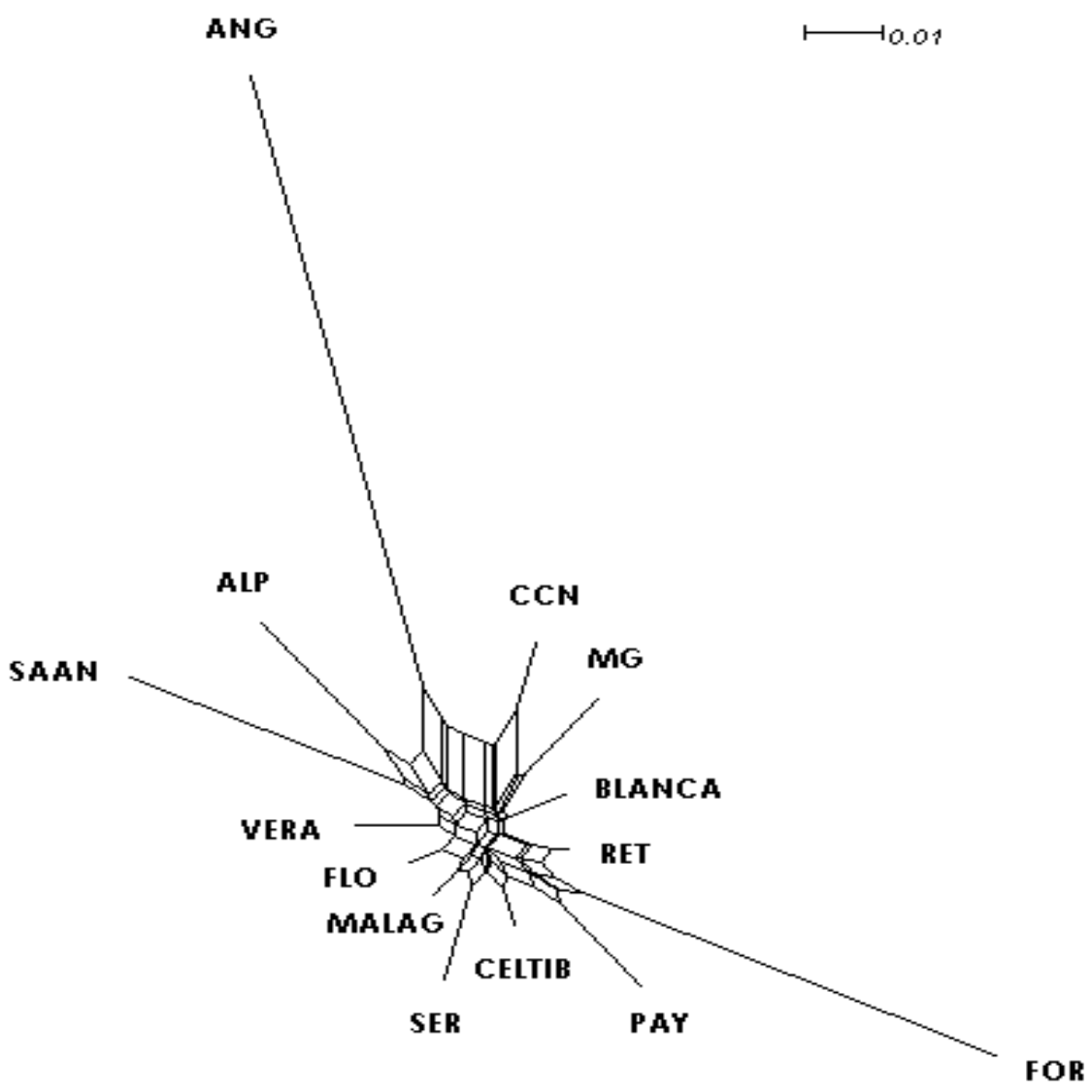

RET $=$ Retinta, VERA = Verata, BLANCA= Blanca serrana, CELTIB $=$ Celtibérica, MALAG= Malagueña, $\mathrm{MG}=$ Murciano granadina, $\mathrm{FLO}=$ Florida, $\mathrm{PAY}=$ Payoya, $\mathrm{SER}=$ Serrana, $\mathrm{FOR}=$ Formentera, $\mathrm{SAAN}=$ Saanen, ALP = Alpina, ANG= Anglonubia, $\mathrm{CCN}=$ Criolla negra.

\section{Análisis de la estructura genética en las poblaciones evaluadas}

El K óptimo para la estructura genética de las poblaciones incluidas en el estudio fue de 9. La representación gráfica del resultado de la estructura genética obtenida de las 14 razas de cabras incluidas en el estudio se presenta en la Figura 2. En la cual, cada individuo está representado por una línea vertical, misma que se divide en segmentos de color que representan la fracción de pertenencia de cada individuo a cada uno de los grupos $(\mathrm{K})$. 
Figura 2: Representación gráfica de la estructura genética de las 14 razas analizadas, asumiendo un número de poblaciones ancestrales K que van de 2 a 9

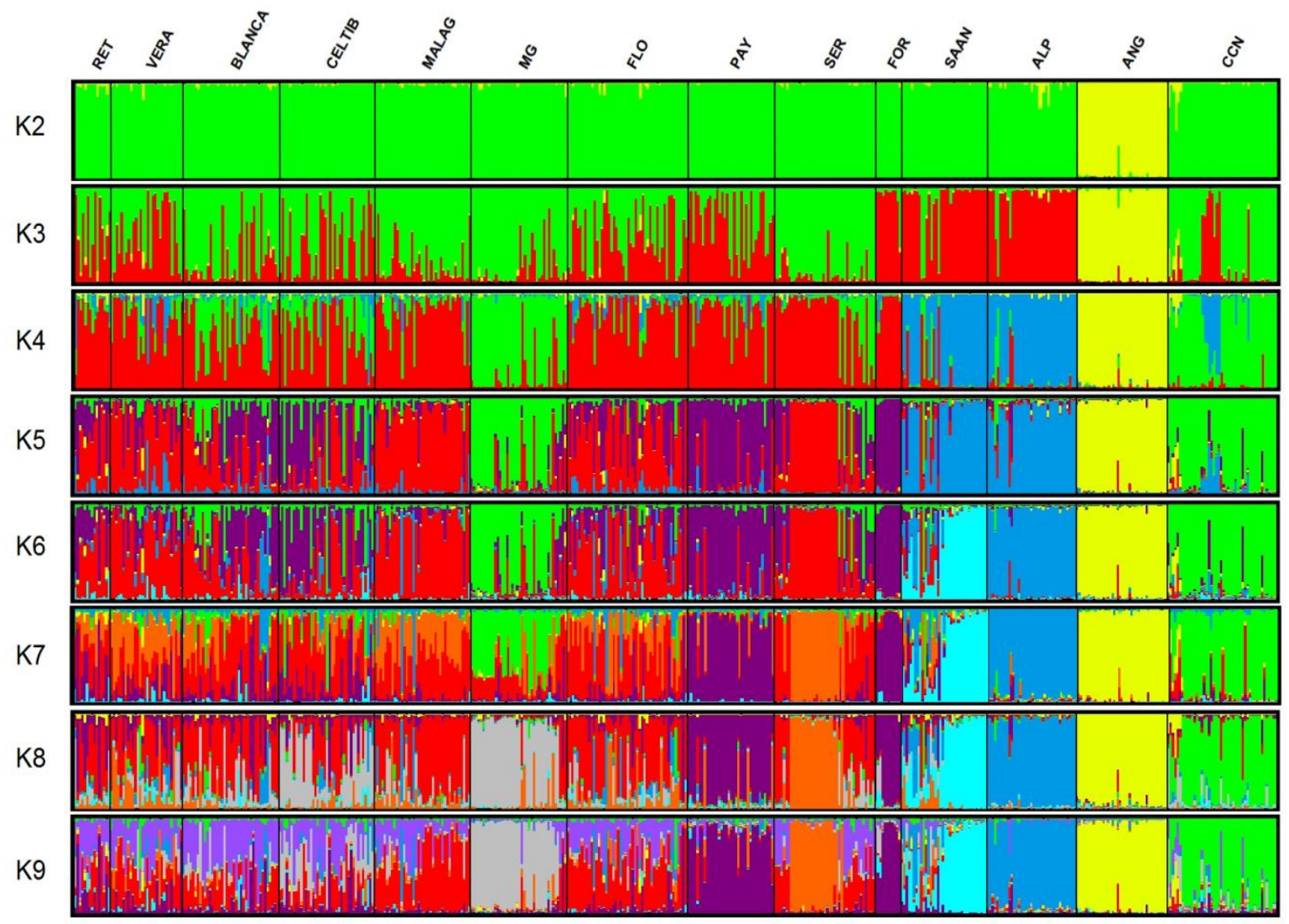

RET $=$ Retinta, VERA= Verata, BLANCA= Blanca serrana, CELTIB= Celtibérica, MALAG= Malagueña, $\mathrm{MG}=$ Murciano granadina, $\mathrm{FLO}=$ Florida, $\mathrm{PAY}=$ Payoya, $\mathrm{SER}=$ Serrana, $\mathrm{FOR}=$ Formentera, $\mathrm{SAAN}=$ Saanen, $\mathrm{ALP}=$ Alpina, $\mathrm{ANG}=$ Anglonubia, $\mathrm{CCN}=$ Criolla negra.

\section{Discusión}

\section{Variación genética en la población Criolla Negra}

Se ha recomendado que los microsatélites utilizados tengan por lo menos cuatro alelos y que el NEA sea superior a dos para ser considerados 7en estudios de diversidad y reducir el error estándar en la estimación de distancias genéticas ${ }^{(38)}$. En el presente estudio solo dos marcadores (MAF209 y SPS115) resultaron con 2 y 3 alelos. Respecto al NEA ETH225 y MAF209 presentaron valores menores a dos (1.41 y 1.25 respectivamente). Lo anterior nos indica que tan apropiados fueron los marcadores para la evaluación de la diversidad genética respecto al NA y NEA. El NMA proporcionó información sobre la diversidad genética de la población de $\mathrm{CCN}$, a mayor número de alelos mayor diversidad y viceversa, el resultado obtenido (8.1) se considera alto si se compara con otros trabajos 
de caracterización como el de la cabra criolla cubana ${ }^{(39)}$, cabras saudíes ${ }^{(19)}$, cabras cachemira en China ${ }^{(40,41)}$ y algunas razas de cabras iraníes ${ }^{(42)}$. Sin embargo, tiene valores cercanos al NMA obtenido para cabras lecheras en Sudáfrica ${ }^{(43)}$. El CIP de los marcadores utilizados reveló un valor promedio (0.66) similar al obtenido en el trabajo de la cabra Retinta extremeña ${ }^{(44)}$.

Otra manera de apreciar la diversidad genética es mediante los valores de heterocigosis, ya que dependen del número y de las frecuencias relativas de los alelos ${ }^{(45)}$. Los valores de heterocigosis promedio obtenidos $(\mathrm{He}=0.71$ y $\mathrm{Ho}=0.62)$ se muestran muy similares a los observados en la cabra Blanca Andaluza ${ }^{(46)}$ y Retinta Extremeña ${ }^{(44)}$, aun cuando esta última presenta un MNA mayor a la CCN. Los valores obtenidos para He y Ho indican un nivel importante de variabilidad genética para la $\mathrm{CCN}$ teniendo en cuenta el estado de reducción de la población en el que se encuentra y donde se esperaría un marcado nivel de consanguinidad. La prueba para el HWE reveló 14 marcadores con desviaciones significativas $(P \leq 0.05)$, lo cual indica que en estos existe un déficit de heterocigosis. El hecho que algunos de estos marcadores se comporten homocigóticos puede deberse a acciones que van desde las condiciones de manejo, como el préstamo de sementales y el poco flujo genético en cada rebaño hasta marcadores que pudieran estar vinculados a rasgos productivos debido a selección enfocada a producción de leche y ganancias de peso sin importar relaciones de parentesco ${ }^{(47)}$.

\section{Diferenciación genética entre poblaciones}

Tomando en cuenta que la estimación de FIS y FIT pueden variar de 1 a -1 y que valores positivos indican una deficiencia de heterocigotos y valores negativos un exceso. Los resultados obtenidos para ambos índices $\left(\mathrm{F}_{\text {IS }}=0.067\right.$ y $\left.\mathrm{F}_{\text {IT }}=0.121\right)$ indican que algunos de los marcadores en las razas resultaron homocigotos. Aun cuando los valores son cercanos a cero, estos indican un posible apareamiento entre parientes, resultado que concuerda con lo observado en cabras autóctonas de China $^{(48)}$, India ${ }^{(47)}$, España y Portugal ${ }^{(24)}$. El FST indicó que el $94.2 \%$ de la variabilidad genética en las razas estudiadas se debía a diferencias entre los individuos dentro de la raza y el 5.8\% a diferencias genéticas entre las razas. Este efecto se muestra de forma parecida con el coeficiente de diferenciación genética $\left(\mathrm{G}_{\mathrm{ST}}=0.073\right)$ que indicó que el $7.3 \%$ de la diversidad genética total se dividió entre las razas y por consiguiente la mayor parte de la variabilidad se encontró dentro de las poblaciones con un porcentaje del $92.7 \%$. La diferencia obtenida entre ambos indicadores se debe a que F $_{\text {ST }}$ refleja las propiedades de la distribución de las frecuencias alélicas entre las poblaciones, mientras que GST se define en términos de las frecuencias de la población ${ }^{(49)}$. Los valores obtenidos de FST $_{\text {y }} \mathrm{G}_{\mathrm{ST}}$ hacen referencia al nivel de variación genética que se ha mantenido en las razas incluidas en el estudio. Porcentajes similares a los obtenidos en el presente estudio se observaron para otras poblaciones $\operatorname{caprinas}^{(17,42,50)}$. 


\section{Distancia genética entre poblaciones y su representación gráfica}

El dendrograma Neighbor-Net mostró que las razas caprinas españolas incluidas en el estudio (Retinta, Verata, Blanca Serrana, Celtibérica, Malagueña, Murciano-Granadina, Florida, Payoya, Serrana, Formentera), se mantienen agrupadas, este efecto se ha observado también en otros trabajos ${ }^{(51)}$ y se debe a la estrecha relación genética y geográfica que guardan estas razas. Las razas Saanen y Alpina también se visualizaron agrupadas hacia un extremo del dendrograma, sin embargo, es notoria la distancia genética obtenida para la raza Anglonubia. Esta relación también se pudo observar en una comparación entre estas razas y cabras brasileñas ${ }^{(52)}$, dicho efecto puede atribuirse a una mayor distancia genética con las razas comparadas y no precisamente a relaciones de origen o parentesco. Sin embargo, hay que tomar en cuenta que los animales de cada población han sido seleccionados también en base a sus características morfológicas. Las estimaciones obtenidas de la distancia genética de Reynolds también mostraron que la distancia más corta para la CCN se encuentra en relación con la raza MG (0.038), este resultado sugiere la posible relación genética entre ambas razas.

\section{Análisis de la estructura genética en las poblaciones evaluadas}

Con el análisis de la estructura genética se evaluó el grado de relación entre las diferentes poblaciones, detectando diferencias entre razas por medio del $\mathrm{K}$ óptimo $(\mathrm{K}=9)$. El análisis indica por un lado que la $\mathrm{CCN}$ no muestra signos de cruzamientos con el resto de las razas incluidas en el estudio y por otro lado se evaluó el grado de relación entre las diferentes poblaciones. La diferenciación genética de la población ANG, que se separa desde K2 y se mantiene separada en todos los supuestos debido a que presenta las distancias genéticas mayores, es similar a lo obtenido con la estructura poblacional en cabras criollas de las Américas ${ }^{(53)}$. Este resultado hace notar que la población ANG mantiene su estructura genética con un bajo nivel de mezcla de individuos de las otras 13 poblaciones evaluadas. En las razas españolas incluidas en el estudio, se presentó una estructura genética con más individuos entremezclados, muy similar a lo observado en un estudio de biodiversidad caprina $^{(24)}$. Esto pudiera deberse a que son poblaciones geográficamente cercanas, lo que facilita la migración de individuos de otras poblaciones. Respecto a la CCN, ésta se observa similar a la cabra MG lo que podría confirmar el supuesto origen de la población. Sin embargo, en el K óptimo (K9) la población CCN presenta una estructura totalmente diferente a la raza $\mathrm{MG}$ y al resto de las poblaciones incluidas en el estudio. Este resultado sustenta lo observado en el dendrograma Neighbor-Net y sugiere que la CCN mantiene 
una estructura racial única y diferenciada de poblaciones que pudieron participar en su origen.

\section{Conclusiones e implicaciones}

El presente trabajo presenta los primeros resultados sobre la diversidad y estructura genética de una población de CCN. Los resultados sugieren que esta población presenta cierto grado de diversidad genética dado su nivel de polimorfismo encontrado en la población. Las distancias genéticas de la CCN con respecto a las otras razas incluidas en el estudio, indican que esta población se encuentra claramente diferenciada de las anteriores, motivo por el cual deberá considerarse como una raza de cabras mexicana. La reducida distancia obtenida entre MG y CCN sugiere que ambas poblaciones mantienen un ancestro en común, muy seguramente la raza Granadina. Se puede concluir que la cabra Criolla Negra presenta una estructura racial definida y diferenciada de las razas que pudieron darle origen, siendo ésta la primera raza de cabras mexicanas dentro del mosaico del centro del país descrita desde el punto de vista genético.

\section{Literatura citada:}

1. Naderi S, Rezaeli HR, Taberlet P, Zundel S, Rafat S, Naghash H, et al. Large-Scale Mitochondrial DNA Analysis of the domestic goat reveals six haplogroups with high diversity. PLoS One 2007;2:1-12.

2. Zeder MA, Hesse B. The initial domestication of goats (Capra hircus) in the Zagros Mountains 10,000 years ago. Science 2000;287:2254-2257.

3. Nomura K, Yonezawa T, Mano S, Kawakami S, Shedlock AM, Hasegawa M, et al. Domestication process of the goat revealed by an analysis of the nearly complete mitochondrial protein-encoding genes. PLoS One 2013;8:1-15.

4. Luikart G, Gielly L, Excoffier L, Vigne J, Bouvet J, Taberlet P. Multiple maternal origins and weak phylogeographic structure in domestic goats. Proc Natl Acad Sci USA 2001;98:5927-5932.

5. Rodero A, Delgado JV, Rodero E. Primitive andalusian livestock and their implications in the discovery of America. Arch Zootec 1992;41:383-400.

6. FAO. Statistics Database. FAOSTAT. 2017 (Revisado 2017/12/15). Disponible en: www.fao.org/faostat/es/\#data/QA.

7. Peacock C, Sherman DM. Sustainable goat production-Some global perspectives. Small Ruminant Res 2010;89:70-80. 
8. FAO. La situación de los recursos zoogenéticos mundiales para la alimentación y la agricultura. 2010. Disponible en: http://www.fao.org/docrep/012/a1250s/ a1250s00.htm

9. SAGARPA. Informe sobre la Situación de los Recursos Genéticos Pecuarios en México. 2002. Disponible en: http://www.sagarpa.gob.mx/ganaderia/Publicaciones/ Paginas/InfoRGPecuariosM.aspx

10. Montaldo HH, Meza-Herrera CA. Genetic goat resources in Mexico: Bioeconomical efficiency of local and specialized genotypes. Wool Technol Sheep Breed 1999;47:184-198.

11. Montaldo H, Jutiezb A, Berruecosc JM, Sinchezd F. Performance of local goats and their backcrosses with several breeds in Mexico. Small Ruminant Res 1995;16:97105 .

12. Montaldo H, Tapia G, Juárez A. Algunos factores genéticos y ambientales que influyen sobre la producción de leche y el intervalo entre partos en cabras. Tec Pecu Mex1981;41:32-44.

13. Montaldo H, Torres-Hernández G, Valencia-Posadas M. Goat breeding research in Mexico. Small Ruminant Res 2010;89:155-163.

14. FAO. Molecular genetic characterization of animal genetic resources. FAO Anim Prod Heal Guid. Rome; 2011.

15. Baumung R, Simianer H, Hoffmann I. Genetic diversity studies in farm animals - A survey. J Anim Breed Genet 2004;121:361-373.

16. Jamil T, Antonella A, Philippe B, Roswitha B, Albano B, Marco B, et al. Genetic diversity of Albanian goat breeds based on microsatellite markers. Arch Zootec 2011;60:607-615.

17. Li JY, Chen H, Lan XY, Kong XJ, Min LJ. Genetic diversity of five Chinese goat breeds assessed by microsatellite markers. Czech J Anim Sci 2008;53:315-319.

18. Thuy LT, Binh D Van, Binh NT, Minh LQ, Thuy TTT, Ton ND, et al. Evaluation of genetic diversity and structure of Vietnamese goat populations using multi locus microsatellite markers. Small Ruminant Res 2017;148:43-50.

19. Al-Atiyat RM, Alobre MM, Aljumaah RS, Alshaikh MA. Microsatellite based genetic diversity and population structure of three Saudi goat breeds. Small Ruminant Res 2015;130:90-94.

20. Rout PK, Joshi MB, Mandal A, Laloe D, Singh L, Thangaraj K. Microsatellite-based phylogeny of Indian domestic goats. BMC Genet 2008;9:11.

21. Mello A, Facioni S, Silva C, Sávio P, Teixeira M, Mendeiros T. Paternity in Brazilian goats through the use of DNA microsatellites. Rev Bras Zootec 2010;39:1011-1014. 
22. BioGoat Consortium. Proyecto Biodiversidad Caprina Iberoamericana. Disponible en: https://biogoat.jimdo.com/

23. Walsh PS, Metzger DA, Higuchi R. Chelex 100 as a medium for simple extraction of DNA for PCR-based typing from forensic material. Biotechniques 2013;54:134139.

24. Martínez AM, Gama LT, Delgado JV, Cañón J, Amills M, Bruno de Sousa C, et al. The Southwestern fringe of Europe as an important reservoir of caprine biodiversity. Genet Sel Evol 2015;47:86.

25. Nei M. Analysis of gene diversity in subdivided populations. Proc Nat Acad Sci 1973;70:3321-3323.

26. Botstein D, White RL, Skolnick M, Davis RW. Construction of a genetic linkage map in man using restriction fragment length polymorphisms. Am J Hum Genet 1980;32:314-331.

27. Park SDE. Trypanotolerance in West African cattle and the population genetic effects of selection [Ph.D. Thesis]. Dublin, Ireland: University of Dublin; 2001.

28. Kimura M, Crow JF. The number of alleles that can be maintained in a finite population. Genetics 1964;49:725-738.

29. Raymond M, Rousset F. GENEPOP (Version 1.2): Population genetics software for exact tests and Ecumenicism. J Hered 1995;86:248-249.

30. Nei M. F-statistics and analysis of gene diversity in subdivided populations. Ann Hum Genet 1977;41:225-233.

31. Belkhir K, Borsa P, Chikhi L, Raufaste N, Bonhomme F. GENETIX 4.05, logiciel sous Windows TM pour la génétique des populations. Laboratoire Génome, Populations, Interactions, CNRS UMR 5000, Université de Montpellier II, Montpellier (France). 1996.

32. Reynolds J, Weir BS, Cockerham CC. Estimation of the coancestry coefficient: basis for a short-term genetic distance. Genetics 1983;105:767-779.

33. Huson DH, Bryant D. Application of phylogenetic networks in evolutionary studies. Mol Biol Evol 2006;23:254-267.

34. Pritchard JK, Stephens M, Donnelly P. Inference of population structure using multilocus genotype data. Genetics 2000;155:945-959.

35. Rosenberg NA. DISTRUCT: a program for the graphical display of population structure. Mol Ecol 2004;4:137-138. 
36. Evanno G, Regnaut S, Goudet J. Detecting the number of clusters of individuals using the software STRUCTURE: a simulation study. Mol Ecol 2005;14:26112620 .

37. Earl DA, Bridgett M. STRUCTURE HARVESTER : a website and program for visualizing STRUCTURE output and implementing the Evanno method. Conserv Genet Resour 2012;4:359-361.

38. Barker JSF. A global protocol for determining genetic distances among domestic livestock breeds. 5th World Cong Genet Appl Livest Prod. Canadá; 1994:501-508.

39. Chacón E, Martínez A, La OM, Velázquez FJ, Pérez E, Vicente-Delgado J. Caracterización genética de la cabra Criolla Cubana mediante marcadores microsatélites. Rev Cub Cienc Agr 2010;44:221-226.

40. Di R, Farhad Vahidi SM, Ma YH, He XH, Zhao QJ, Han JL, et al. Microsatellite analysis revealed genetic diversity and population structure among Chinese cashmere goats. Anim Genet 2011;42:428-431.

41. Du X, Cao J, Han X, Hao H, Yu M, Zhang G, et al. Genetic diversity and population structure among eight Chinese indigenous goat breeds in the Yellow River valley. Small Ruminant Res 2017;148:87-92.

42. Vahidi SMF, Tarang AR, Naqvi AN, Falahati Anbaran M, Boettcher P, Joost S, et al. Investigation of the genetic diversity of domestic Capra hircus breeds reared within an early goat domestication area in Iran. Genet Sel Evol 2014;46:1-12.

43. Bosman L, van Marle-Köster E, Visser C. Genetic diversity of South African dairy goats for genetic management and improvement. Small Ruminant Res 2015;123:224-231.

44. Parejo JC, Padilla JA, Calero R, Martínez-Trancón M, Sansinforiano E, Rabasco A, et al. Population genetic structure and conservation management of Retinta Extremeña goats. Small Ruminant Res 2015;124:9-16.

45. Hale ML, Burg TM, Steeves TE. Sampling for microsatellite-based population genetic studies: 25 to 30 individuals per population is enough to accurately estimate allele frequencies. PLoS One 2012;7:1-10.

46. Martínez AM, Carrera MP, Acosta JM, Rodríguez-Gallardo PP, Cabello A, Camacho $\mathrm{E}$, et al. Genetic characterization of the Blanca Andaluza goat based on microsatellite markers. South African J Anim Sci 2004;34:17-19.

47. Dixit SP, Verma NK, Aggarwal RAK, Vyas MK, Rana J, Sharma A. Genetic diversity and relationship among Indian goat breeds based on microsatellite markers. Small Ruminant Res 2012;105:38-45. 
48. Cao J, Li X, Du X, Zhao S. Microsatellite based genetic diversity and population structure of nine indigenous Chinese domestic goats. Small Ruminant Res 2017; 148:80-86.

49. Holsinger KE, Weir BS. Genetics in geographically structured populations: defining, estimating and interpreting Fst. Nat Rev Genet 2009;10:639-650.

50. Martínez AM, Acosta J, Vega-Pla JL, Delgado JV. Analysis of the genetic structure of the canary goat populations using microsatellites. Livest Sci 2006;102:140-145.

51. Cañón J, García D, García-Atance MA, Obexer-Ruff G, Lenstra JA, Ajmone-Marsan $\mathrm{P}$, et al. Geographical partitioning of goat diversity in Europe and the Middle East. Anim Genet 2006;37:327-334.

52. Oliveira JD De, Luiza M, Paiva S De, Mírian T, Machado M, Miretti MM, et al. Structure and genetic relationships between Brazilian naturalized and exotic purebred goat domestic goat (Capra circus) breeds based on microsatellites. Genet Mol Biol 2007;30:356-363.

53. Ginja C, Gama LT, Mart A, Sevane N, Lanari MR. Genetic diversity and patterns of population structure in Creole goats from the Americas. Anim Genet 2017;1-15. 